\title{
An Implementation and Evaluation of the AMLS Method for Sparse Eigenvalue Problems*
}

\author{
Weiguo $\mathrm{Gao}^{\dagger} \quad$ Xiaoye S. $\mathrm{Li}^{\ddagger} \quad$ Chao Yang ${ }^{\ddagger} \quad$ Zhaojun Bai ${ }^{\S}$
}

\begin{abstract}
We describe an efficient implementation and present a performance study of an algebraic multilevel sub-structuring (AMLS) method for sparse eigenvalue problems. We assess the time and memory requirements associated with the key steps of the algorithm, and compare it with the shift-and-invert Lanczos algorithm in computational cost. Our eigenvalue problems come from two very different application areas: the accelerator cavity design and the normal mode vibrational analysis of the polyethylene particles. We show that the AMLS method, when implemented carefully, is very competitive with the traditional method in broad application areas, especially when large numbers of eigenvalues are sought.
\end{abstract}

\section{Introduction}

The automated multilevel sub-structuring (AMLS) method $[2,9,3]$ is a multi-level extension of a simple sub-structuring method called component mode synthesis (CMS) [8, 4] originally developed in the 1960s for solving eigenvalue problems arising from structure engineering analysis. The method has recently been shown to be efficient for performing vibration and acoustic analysis of large scale finite element models of automobile bodies [9] on a workstation. In particular, it was shown in [12] that AMLS is several times faster than the shift-invert Lanczos (SIL) algorithm commonly used in structure engineering.

The efficiency exhibited by AMLS can be attributed to two main factors: 1) The method, which can be characterized as a projection method, does not construct or maintain an orthornormal basis of the subspace into which the original eigenvalue problem is projected. This feature makes AMLS extremely appealing when the number of desired eigenvalues is large. 2) The method does not perform a sequence of triangular substitutions required in SIL. Thus, it is suitable for an out-ofcore implementation that requires a limited amount of input and output (I/O) traffic.

It is important to recognize that AMLS usually produces approximate solutions that are less accurate than those computed by SIL. Although the lack of accuracy can be tolerated in some applications such as frequency response analysis, it may be a cause for concern in others. There are a number of ways to improve the accuracy of the AMLS algorithm [1]. However, these improvements

${ }^{*}$ This work was supported by the Director, Office of Advanced Scientific Computing Research, Division of Mathematical, Information, and Computational Sciences of the U.S. Department of Energy under contract number DEAC03-76SF00098.

†School of Mathematical Sciences, Fudan University, Shanghai 200433, China. wggao@fudan.edu.cn. The work of this author was performed while he was visiting Lawrence Berkeley National Laboratory.

${ }^{\ddagger}$ Computational Research Division, Lawrence Berkeley National Laboratory, Berkeley, CA 94720. \{xsli, cyang\}@lbl.gov.

${ }^{\S}$ Department of Computer Science, University of California, Davis, CA 95616. bai@cs.ucdavis.edu. 
tend to increase the runtime of the computation significantly. Therefore, when we measure the performance of AMLS and compare it with other techniques, we should keep the accuracy issue in perspective.

As we will see in Section 2, the basic AMLS algorithm is conceptually easy to describe. However, an efficient implementation of the algorithm would require matrix transformations to be carefully organized to minimize the number of floating point operations and the amount of memory usage. A number of decisions have to be made at different stages of the algorithm to balance the cost and accuracy of the computation. These decisions will obviously affect the outcome and performance of the calculation.

In this paper, we examine a number of practical issues arising from the implementation of the AMLS algorithm. We will evaluate the performance of AMLS through two examples that are not related to structural analysis. Our performance evaluation is based on runtime, memory usage, and accuracy. To the best of our knowledge, this type of empirical analysis of the AMLS method has not been performed outside of structure analysis applications.

We show that AMLS is more efficient than the traditional shift-and-invert Lanczos method when a large number of eigenvalues are wanted and when the desired accuracy is limited to a few digits. Instead of performing the matrix transformations required in AMLS out-of-core, we developed an in-core implementation that requires a significantly less amount of memory than a straightforward implementation. The key idea behind such an implementation is to recompute some of the intermediate matrix blocks instead of storing them (either in memory or on a disk). We will show that such an implementation of AMLS results in up to $50 \%$ of memory saving while incurring less than $15 \%$ increase in runtime.

Our paper is organized as follows. In Section 2, we give a brief overview on the algorithmic ingredients of the AMLS method. We also discuss the accuracy of the algorithm and examine how the selection of sub-structure eigenvectors (modes) affect the quality of the approximate eigenpairs. The implication of mode selection on the implementation will also be discussed. In Section 3 , we examine the implementation details. In particular, we illustrate how the matrix operations in AMLS can be organized to reduce both floating point operations and memory usage. Our discussion makes use of a graph-theoretical tool called seperator tree that allows one to easily track the data flow and task dependency in the sub-structuring computation. We also present a memory saving technique that requires some intermediate results to be recomputed instead of stored. In Section 4, we evaluate the performance of AMLS on two problems arising from two different application domains.

\section{The algorithmic view of AMLS}

Before we get into the nitty gritty details of the implementation issues, it is instructive to review the basic idea behind the AMLS algorithm for solving the following algebraic eigenvalue problem

$$
K x=\lambda M x,
$$

where $K$ is symmetric and $M$ is symmetric positive definite, and both are sparse and of dimension $n$-by- $n$. They may or may not have the same sparsity pattern. Our description of the method does not make use of any information regarding the geometry or the physical structure on which the original problem is defined. Therefore, it is purely algebraic. 


\subsection{A single-level algorithm}

Let the rows and columns of $K$ and $M$ be permuted so that these matrices can be partitioned as

$$
K={ }_{n_{2}}^{n_{1}}{ }_{n_{3}}\left(\begin{array}{ccc}
n_{1} & n_{2} & n_{3} \\
K_{11} & & K_{13} \\
& K_{22} & K_{23} \\
K_{13}^{T} & K_{23}^{T} & K_{33}
\end{array}\right) \text { and } M={ }_{n_{2}}^{n_{1}}\left(\begin{array}{ccc}
M_{11} & n_{2} & n_{3} \\
& M_{22} & M_{23} \\
M_{13}^{T} & M_{23}^{T} & M_{33}
\end{array}\right)
$$

where the labels $n_{1}, n_{2}$ and $n_{3}$ indicate the dimensions of the submatrix blocks, and $n_{1}+n_{2}+n_{3}=n$. This permutation can be obtained by applying a matrix ordering and partitioning algorithm such as the nested dissection algorithm [7] to the structure of the matrix $|K|+|M|$ (ignoring numerical cancellation).

The pencils $\left(K_{11}, M_{11}\right)$ and $\left(K_{22}, M_{22}\right)$ now define two algebraic sub-structures that are connected by the third block rows and columns of $K$ and $M$ which is referred to as the interface block. We assume that $n_{3}$ is much smaller than $n_{1}$ and $n_{2}$.

In a single-level algebraic sub-structuring algorithm, we proceed by performing a block factorization

$$
K=L D L^{T}
$$

where

$$
L=\left(\begin{array}{ccc}
I_{n_{1}} & & \\
& I_{n_{2}} & \\
K_{13}^{T} K_{11}^{-1} & K_{23}^{T} K_{22}^{-1} & I_{n_{3}}
\end{array}\right) \quad \text { and } D=\left(\begin{array}{ccc}
K_{11} & & \\
& K_{22} & \\
& & \widehat{K}_{33}
\end{array}\right)
$$

The last diagonal block of $D$, often known as the Schur complement, is defined by

$$
\widehat{K}_{33}=K_{33}-K_{13}^{T} K_{11}^{-1} K_{13}-K_{23}^{T} K_{22}^{-1} K_{23} .
$$

The inverse of the lower triangular factor $L$ defines a congruence transformation that, when applied to the matrix pencil $(K, M)$, yields a new matrix pencil $(\widehat{K}, \widehat{M})$ :

$$
\widehat{K}=L^{-1} K L^{-T}=D \quad \text { and } \quad \widehat{M}=L^{-1} M L^{-T}=\left(\begin{array}{ccc}
M_{11} & & \widehat{M}_{13} \\
& M_{22} & \widehat{M}_{23} \\
\widehat{M}_{13}^{T} & \widehat{M}_{23}^{T} & \widehat{M}_{33}
\end{array}\right) .
$$

The off-diagonal blocks of $\widehat{M}$ satisfy

$$
\widehat{M}_{i 3}=M_{i 3}-M_{i i} K_{i i}^{-1} K_{i 3}, \text { for } i=1,2 .
$$

The last diagonal block of $\widehat{M}$ satisfies

$$
\widehat{M}_{33}=M_{33}-\sum_{i=1}^{2}\left(K_{i 3}^{T} K_{i i}^{-1} M_{i 3}+M_{i 3}^{T} K_{i i}^{-1} K_{i 3}-K_{i 3}^{T} K_{i i}^{-1} M_{i i} K_{i i}^{-1} K_{i 3}\right) .
$$

The pencil $(\widehat{K}, \widehat{M})$ is often known as the Craig-Bampton form [4] in structure engineering. Note that the eigenvalues of $(\widehat{K}, \widehat{M})$ are identical to those of $(K, M)$, and the corresponding eigenvectors $\widehat{x}$ are related to the eigenvectors of the original problem (1) through $\widehat{x}=L^{T} x$. 
The sub-structuring algorithm constructs a subspace in the form of

$$
S={ }_{n_{2}}^{n_{1}} \begin{array}{ccc}
k_{1} & k_{2} & k_{3} \\
n_{3}
\end{array}\left(\begin{array}{lll}
S_{1} & & \\
& S_{2} & \\
& & S_{3}
\end{array}\right)
$$

where $S_{1}, S_{2}$ and $S_{3}$ consist of $k_{1}, k_{2}$ and $k_{3}$ selected eigenvectors of $\left(K_{11}, M_{11}\right),\left(K_{22}, M_{22}\right)$ and $\left(\widehat{K}_{33}, \widehat{M}_{33}\right)$ respectively. The eigenvectors associated with $\left(K_{11}, M_{11}\right)$ and $\left(K_{22}, M_{22}\right)$ will be referred to as the sub-structure modes, and those associated with $\left(\widehat{K}_{33}, \widehat{M}_{33}\right)$ will be referred to as the coupling modes. The approximation to the desired eigenpairs of the pencil $(\widehat{K}, \widehat{M})$ are obtained by projecting the pencil $(\widehat{K}, \widehat{M})$ onto the subspace spanned by $S$, i.e., we seek $\theta$ and $q \in \mathbb{R}^{k_{1}+k_{2}+k_{3}}$ such that

$$
\left(S^{T} \widehat{K} S\right) q=\theta\left(S^{T} \widehat{M} S\right) q
$$

It follows from the standard Rayleigh-Ritz theory [15, page 213] that $\theta$ is the best approximation to an eigenvalue of $(K, M)$ from $\operatorname{span}\{S\}$, and the vector formed by $z=L^{-T} S q$ gives an approximation to the corresponding eigenvector.

We make the following remarks. 1) The algorithm is most efficient when $k_{i}$ can be chosen to be much smaller than $n_{i}$. In this case, $S_{i}$ can be computed by a shift-and-invert Lanczos procedure. The cost of this computation is generally small compared to the rest of the computation, especially when this algorithm is extended to a multilevel scheme. 2) In some applications, $n_{3}$ can be much smaller than both $n_{1}$ and $n_{2}$. In this case, one can retain all the coupling modes by simply setting $k_{3}=n_{3}$ and replacing $S_{3}$ with $I_{n_{3}}$.

Decisions must be made on how to select eigenvectors from each sub-structure. The selection should be made in a way so that the dimension of the subspace spanned by columns of $S$ is small while a sufficient amount of spectral information of $(K, M)$ is retained. The process of choosing appropriate eigenvectors from each sub-structure is referred to as mode selection [16]. A few strategies have been proposed in [6] on how to select eigenvectors from each sub-structure. However, all of these stategies, which are heuritics derived from the the accuracy analysis of the substructuring algorithm, tend to be somewhat conservative, i.e. they tend to select more eigenvectors than is necessary. More research is required to develop better strategies to optimize the performance of the sub-structuring algorithm.

\section{$2.2 \quad$ A multi-level extension}

The single-level algebraic sub-structuring algorithm can be extended to a multilevel algorithm in a natural way. The matrix reordering and partitioning scheme used to create the block structure (2) can be applied recursively to $\left(K_{11}, M_{11}\right)$ and $\left(K_{22}, M_{22}\right)$ respectively to produce a multilevel division of $(K, M)$ into smaller submatrices. We will use a two-level example below to illustrate the major steps of the multi-level algorithm.




Because of symmetry, we store only the non-zero matrix elements in the lower triangular portions of $K$ and $M$. This multilevel nested structure can be obtained by a graph partitioning software package such as METIS [10] which algebraically identifies the separators recursively. In addition to providing an ordering scheme, METIS also returns a separator tree, a hierarchical graph in which each node is mapped to a set of columns (or rows) in $K$ and $M$. In a separator tree such as the one shown in Figure 1(a), the top level node (the rectangular box labeled with 15) corresponds to the first-level separator that partitions $K$ and $M$ into two sub-structures. In Figure 1(b), this separator is shown as shaded rows and columns that intersect at the lower right corner of the matrix marked by 15. Each sub-structure can be further partitioned into two smaller sub-structures. The separators associated with these sub-partitions (marked by 7 and 14 in Figure 1(a) and Figure 1(b)) are connected to the first level separator in Figure 1(a) so that the hierarchical structure of the partition can be identified easily. A sub-structure that is not further partitioned is represented by a leaf node that appears at the bottom of the separator tree. In addition to characterizing the nested structure of a matrix partition, the separator tree can also be used succinctly to describe the computational tasks and their dependencies in the AMLS algorithm as we will see below.

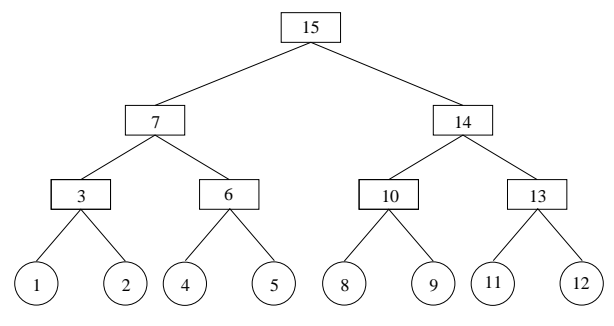

(a)

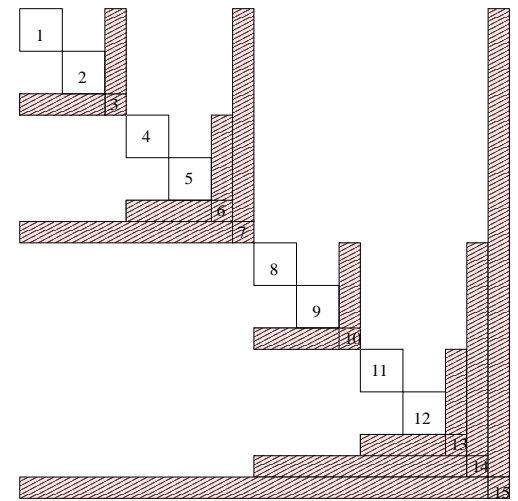

(b)

Figure 1: The separator tree (a) and the reordered matrix (b) for a three-level dissection.

Once the matrix pencil $(K, M)$ has been partitioned and reordered in the form of $(7)$, we can perform Gaussian elimination on $K$ to obtain $K=L D L^{T}$, where $D$ is block diagonal as follows

$$
D=L^{-1} K L^{-T}=\operatorname{diag}\left(K_{11}, K_{22}, \widehat{K}_{33}, K_{44}, K_{55}, \widehat{K}_{66}, \widehat{K}_{77}\right) \stackrel{\text { def }}{=} \widehat{K} .
$$

In this elimination process, only the diagonal blocks corresponding to the separators (i.e., $K_{33}, K_{66}$, and $\left.K_{77}\right)$ are modified. The diagonal blocks corresponding to the leaves are not altered. Applying the same congruence transform to $M$ yields



Note that $\widehat{M}$ and $M$ have the same block structure. Again, the diagonal blocks associated with the separator tree leaves are not altered, but all other blocks (including all the off-diagonal blocks) 
are modified. Moreover, the off-diagonal blocks of $\widehat{M}$ typically contain more non-zeros than the corresponding blocks in $M$.

The congruence tranformation applied to $(K, M)$ is followed by several independent sub-structure calculations in which selected eigenpairs of the smaller pencils $\left(K_{i i}, M_{i i}\right)$ are computed for $i=$ $1,2, \ldots, N$, where $N$ is the number of leaf nodes in the separator tree. Let $S_{i}$ be the matrix that contains $k_{i}$ computed eigenvectors of $\left(K_{i i}, M_{i i}\right)$ (or $\left(\widehat{K}_{i i}, \widehat{M}_{i i}\right)$ ) as its columns. These eigenvectors are assembled to form an $n$-by- $p$ matrix

$$
S=\operatorname{diag}\left(S_{1}, S_{2}, \ldots, S_{N}\right)
$$

where $p=\sum_{i=1}^{N} k_{i}$. Approximate eigenpairs of $(K, M)$ can then be obtained by applying the Rayleigh-Ritz procedure within the subspace $\operatorname{span}\{S\}$. To summarize, we list the major steps of the AMLS algorithm in Algorithm 1.

\section{Algorithm 1 Algebraic Multi-level Sub-structuring (AMLS)}

Input: $\quad$ A matrix pencil $(K, M)$, where $K$ is symmetric and nonsingular and $M$ is symmetric positive definite

Output: $\quad \theta_{j} \in R^{1}$ and $z_{j} \in R^{n},(j=1,2, \ldots, k)$ such that $K z_{j} \approx \theta_{j} M z_{j}$

(1) Partition and reorder $K$ and $M$ to be in the form of (7)

(2) Perform block factorization $K=L D L^{T}$

(3) Apply the congruence transformation defined by $L^{-1}$ to $(K, M)$ to obtain $(\widehat{K}, \widehat{M})$ defined by $(8)$ and $(9)$

(4) Compute a subset of the eigenpairs of interest for the subproblems $\left(K_{i i}, M_{i i}\right)$ (and $\left.\left(\widehat{K}_{i i}, \widehat{M}_{i i}\right)\right)$. Then, form the matrix $S$ in (10)

(5) $\quad$ Project the matrix pencil $(\widehat{K}, \widehat{M})$ onto the subspace $\operatorname{span}\{S\}$

(6) $\quad$ Compute $k$ desired eigenpairs $\left(\theta_{j}, q_{j}\right)$ from $\left(S^{T} \widehat{K} S\right) q=\theta\left(S^{T} \widehat{M} S\right) q$, and set $z_{j}=L^{-T} S q_{j}$ for $j=1,2, \ldots, k$

(7) Apply the inverse of the permutation used in Step (1) to $z_{j}$ to restore the original order of the solution.

\subsection{Accuracy}

The accuracy of the approximate eigenpairs would depend on how $S_{1}, S_{2}$ and $S_{3}$ are constructed in (5). Intuitively, if one increases the dimension of the subspace $\operatorname{span}\{S\}$ by computing more sub-structure eigenvectors (modes) and including them in $S_{i}$, the accuracy of the approximation can be improved. However, the analysis performed in [16] indicates that the amount of accuracy improvement may be negligible as a result of simply including more sub-structure modes in $S_{i}$ while the cost of solving the projected eigenvalue problem

$$
\left(S^{T} \widehat{K} S\right) q=\theta\left(S^{T} \widehat{M} S\right) q
$$

can become significantly higher.

Thus, before we begin to discuss the implementation of AMLS, it is worthwhile to take a closer look at the relationship between mode selection and the accuracy of the approximate eigenpairs returned by AMLS. The following dissusion is a summary of the analysis presented in [16]. 
To simplify the discussion, we will look at a single-level partitioning, and work with the matrix pencil $(\widehat{K}, \widehat{M})$, where $\widehat{K}$ and $\widehat{M}$ are defined in (4). As noted earlier, $(\widehat{K}, \widehat{M})$ and $(K, M)$ have the same set of eigenvalues. If $\widehat{x}$ is an eigenvector of $(\widehat{K}, \widehat{M})$, then $x=L^{-T} \widehat{x}$ is an eigenvector of $(K, M)$, where $L$ is the transformation defined in (3).

Let $\left(\mu_{j}^{(i)}, v_{j}^{(i)}\right)$ be the $j$-th eigenpair of the $i$-th subproblem $(i=1,2)$, and $\left(\mu_{j}^{(3)}, v_{j}^{(3)}\right)$ be the $j$-th eigenpair of the coupling subproblem, i.e.,

$$
\begin{aligned}
K_{i i} v_{j}^{(i)} & =\mu_{j}^{(i)} M_{i i} v_{j}^{(i)}, \text { for } i=1,2 \\
\widehat{K}_{i i} v_{j}^{(3)} & =\mu_{j}^{(3)} \widehat{M}_{i i} v_{j}^{(3)}
\end{aligned}
$$

where $\left(v_{j}^{(i)}\right)^{T} M_{i i} v_{k}^{(i)}=\delta_{j, k},\left(v_{j}^{(3)}\right)^{T} \widehat{M}_{i i} v_{k}^{(3)}=\delta_{j, k}$, and

$$
\delta_{j, k}= \begin{cases}1 & \text { if } j=k \\ 0 & \text { otherwise }\end{cases}
$$

Let $\mu_{j}^{(i)}$ be ordered such that

$$
\mu_{1}^{(i)} \leq \mu_{2}^{(i)} \leq \cdots \leq \mu_{n_{i}}^{(i)}
$$

then we can express $\widehat{x}$ as

$$
\widehat{x}=\left(\begin{array}{ccc}
V_{1} & & \\
& V_{2} & \\
& & V_{3}
\end{array}\right)\left(\begin{array}{l}
y_{1} \\
y_{2} \\
y_{3}
\end{array}\right),
$$

where $V_{i}=\left(v_{1}^{(i)} v_{2}^{(i)} \ldots v_{n_{i}}^{(i)}\right), i=1,2,3$, and $y=\left(y_{1}^{T}, y_{2}^{T}, y_{3}^{T}\right)^{T} \neq 0$.

It is easy to verify that $y$ satisfies the following canonical generalized eigenvalue problem

$$
\left(\begin{array}{ccc}
\Lambda_{1} & & \\
& \Lambda_{2} & \\
& & \Lambda_{3}
\end{array}\right)\left(\begin{array}{l}
y_{1} \\
y_{2} \\
y_{3}
\end{array}\right)=\lambda\left(\begin{array}{ccc}
I_{n_{1}} & & G_{13} \\
& I_{n_{2}} & G_{23} \\
G_{13}^{T} & G_{23}^{T} & I_{n_{3}}
\end{array}\right)\left(\begin{array}{l}
y_{1} \\
y_{2} \\
y_{3}
\end{array}\right)
$$

where $\Lambda_{i}=\operatorname{diag}\left(\mu_{1}^{(i)}, \mu_{2}^{(i)}, \ldots, \mu_{n_{i}}^{(i)}\right)$ for $i=1,2,3$, and $G_{i 3}=V_{i}^{T} \widehat{M}_{i 3} V_{3}$ for $i=1,2$. This pencil is clearly congruent to the pencils $(\widehat{K}, \widehat{M})$ and $(K, M)$. Thus it shares the same set of eigenvalues with that of $(K, M)$. If $\widehat{x}$ can be well approximated by a linear combination of the columns of $S$, then the vector $y_{i}(i=1,2,3)$ must contain only a few entries with large magnitude. All the other components of $y_{i}$ are likely to be small and negligible. Multiplying (14) from the left by $\operatorname{diag}\left(\Lambda_{1}, \Lambda_{2}, \Lambda_{3}\right)^{-1}$ yields

$$
\begin{aligned}
y_{i} & =\lambda\left(\Lambda_{i}-\lambda I_{n_{i}}\right)^{-1} G_{i 3} y_{3}, \quad i=1,2 \\
y_{3} & =\lambda\left(\Lambda_{3}-\lambda I_{n_{3}}\right)^{-1}\left(G_{13}^{T} y_{1}+G_{23}^{T} y_{2}\right)
\end{aligned}
$$

It follows that the $j$-th component of $y_{i}$ is given by

$$
\left|e_{j}^{T} y_{i}\right|=\rho_{\lambda}\left(\mu_{j}^{(i)}\right) g_{j}^{(i)},
$$

where,

$$
\begin{array}{r}
\rho_{\lambda}(\omega)=|\lambda /(\omega-\lambda)|, \\
g_{j}^{(i)}=\left|e_{j}^{T} G_{i 3} y_{3}\right| \text { for } i=1,2, \text { and } g_{j}^{(3)}=\left|e_{j}^{T}\left(G_{13}^{T} y_{1}+G_{23}^{T} y_{2}\right)\right| .
\end{array}
$$


When $g_{j}^{(i)}$ can be bounded from above and below by a moderate constant, the magnitude of $\left|e_{j}^{T} y_{i}\right|$ is essentially determined by $\rho_{\lambda}\left(\mu_{j}^{(i)}\right)$ which is called a $\rho$-factor in [16]. It is easy to see that $\rho_{\lambda}\left(\mu_{j}^{(i)}\right)$ is large when $\mu_{j}^{(i)}$ is close to $\lambda$, and it is small when $\mu_{j}^{(i)}$ is away from $\lambda$. For the smallest eigenvalue $\lambda_{1}$ of $(K, M)$, it is easy to show that $\rho_{\lambda_{1}}\left(\mu_{j}^{(i)}\right)$ is monotonically decreasing with respect to $j$. Thus, if $\lambda_{1}$ is the desired eigenvalue, one should naturally choose the matrix $S_{i}$ in (5) to contain only the leading $k_{i}$ columns of $V_{i}$, for some $k_{i} \ll n_{i}$. When more than one eigenpairs are needed, the selection criterion becomes slightly more complicated. It will involve examining the $\rho$-factors associated with the smallest and largest eigenvalues of interest.

It is shown in [16] that a modest upper bound for $g_{j}^{(i)}$ can be easily derived from the orthonormality constraint on the eigenvector of (14). However, it appears to be difficult to derive a lower bound on $g_{j}^{(i)}$. The numerical examples presented in [16] indicates that $g_{j}^{(i)}$ can be tiny for some $\mathrm{j}$ when $\rho_{j}^{(i)}$ is relatively large. In this case, one can potentially leave the eigenvector associated with $j$-th eigenvalue of the $i$-th sub-structure out of $S_{i}$ without sacrificing the accuracy of the approximate eigenpair. This would reduce the cost associated with solving the projected problem (11) in the AMLS algorithm. However, since we cannot compute the magnitude of $g_{j}^{(i)}$ in advance, such a mode selection strategy is not practical. We are currently investigating a number of ways to estimate the magnitude of $g_{j}^{(i)}$, these estimates may be used to improve the existing mode selection strategy which is based merely on a $\rho$-factor threshold.

\section{Implementing AMLS}

We should emphasize that the major steps outlined in Algorithm 1 constitute only a conceptual description of the AMLS procedure. To develop an efficient implementation, one must carefully organize these steps in a way that would lead to minimal use of memory and floating-point operations (flops).

The complexity of the implementation is magnified by the fact that the congruence transformation $L^{-1} M L^{-T}$ involves more matrix operations than a block Gaussian elimination. The update of $M$ must be carried out by multiplying $M$ with block elementary eliminators of $K$ (and their transposes) from both the left and the right. Hence, the computation of $L^{-1} M L^{-T}$ cannot be organized as a strictly left-looking or right-looking procedure; it has both right- and left-looking dataflow pattern.

If we were to compute $\widehat{M}=L^{-1} M L^{-T}$ explicitly as suggested by Step (3) of Algorithm 1, the memory usage of the algorithm would become excessive because $\widehat{M}$ is likely to contain a significant number of non-zero fills in each off-diagonal matrix block. However, since computing the desired eigenvalues and the corresponding eigenvectors does not require the presence of $\widehat{M}$ explicitly, a more efficient scheme is to project $M$ into the subspace spanned by columns of $L^{-T} S$ incrementally as $L$ and $S$ are being computed. In another word, we can interleave the computations of block elimination (Steps (2) and (3)), the computations of the sub-structure eigenvectors (Step (4)), and the formation of the projected matrices (Step (5)). The pseudo-code in Figure 2 illustrates how specific matrix operations performed in Steps (2)-(5) of Algorithm 1 are interleaved. The sequence in which these matrix operations are performed can also be conveniently described in terms of the traversal of the separator tree shown in Figure 1. 


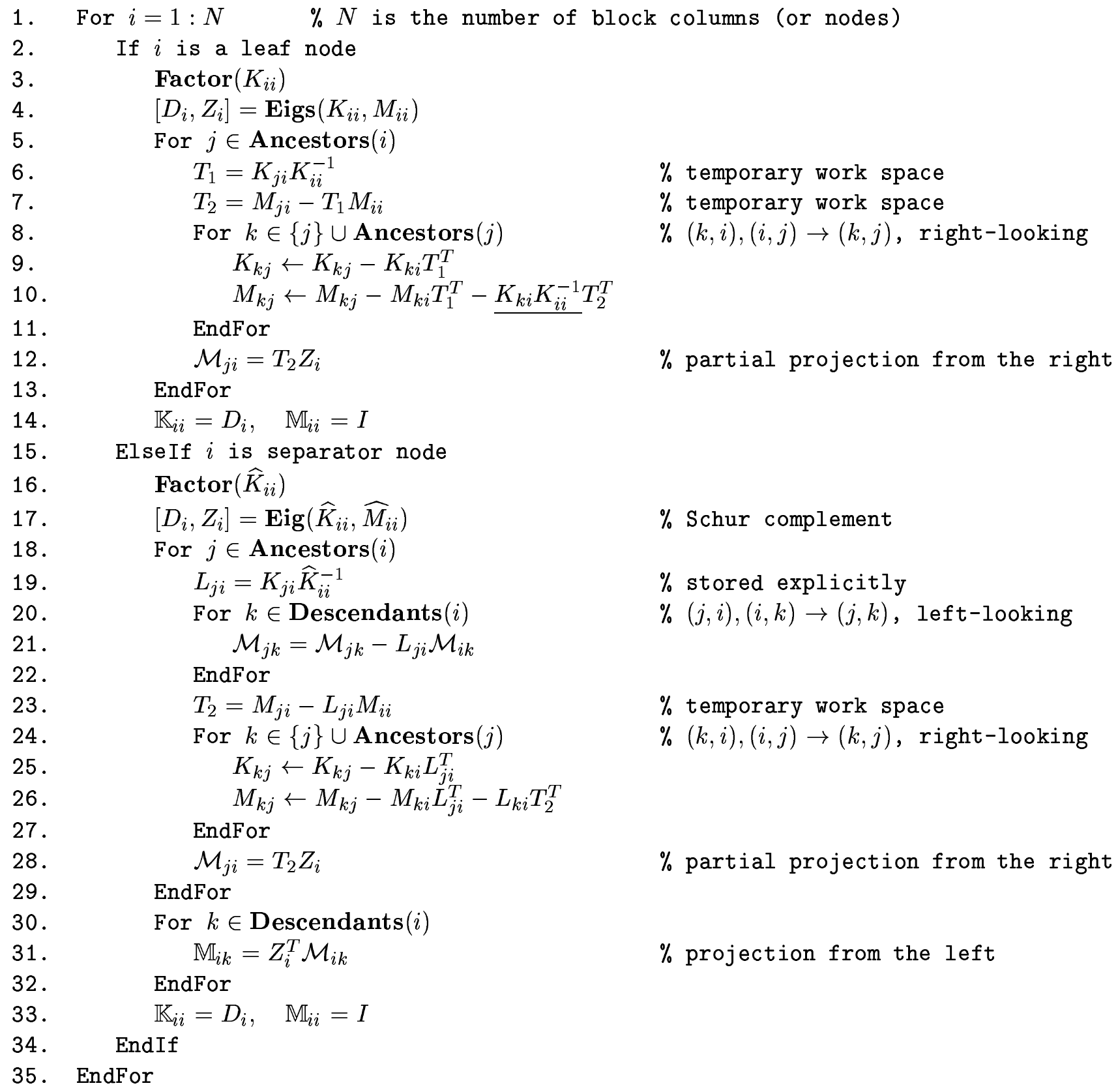

Figure 2: Pseudo-code for Steps (2)-(5) of Algorithm 1. 


\subsection{Interleave congruence transformation and projection calculation}

For a node $i$ in a separator tree, we use $\operatorname{Ancestors}(i)$ to denote the set of nodes on the path from $i$ 's parent to the root of the tree. For instance, Ancestors $(10)=\{14,15\}$ in Figure 1. Similarly, we use Descendants $(i)$ to denote the set of nodes that belong to the subtree rooted at $i$ (excluding i).

We distinguish the operations performed at leaf nodes from those performed at upper level tree nodes. At a leaf node $i$, one performs a Cholesky factorization of the diagonal block $K_{i i}$ in order to construct the $i$-th block column of $L$. Let $m_{i}$ be the dimension of $K_{i i}$. It follows from the standard theory on block Gaussian elimination that computing the $i$-th block column of $L$ defines an elementary block eliminator

$$
L_{e_{i}}=L E_{i} E_{i}^{T}
$$

where $E_{i}=\left(0, \ldots, I_{i}, \ldots, 0\right)^{T}$ is an $n \times m_{i}$ matrix. Note that $L=\Pi_{i=1}^{n_{b}} L_{e_{i}}$, where $n_{b}$ is the number of block columns in $L$. We say that the $i$-th leaf node is eliminated when we mulitply $K$ from the left and right by $L_{e_{i}}^{-1}$ and $L_{e_{i}}^{-T}$ respectively.

Applying $L_{e_{i}}$ to $K$ and $M$ amounts to modifying block rows and columns associated with the tree nodes that belong to Ancestors $(i)$. These nodes are shaded in Figure 3(a), for example, when the leaf node being eliminated is the first block column of $K$ and $M$. The corresponding matrix blocks being updated in $K$ and $M$ are shaded in Figure 3(b). It is important to note that the update of $K$ is "one-sided" (Line 9 of the pseudo-code in Figure 2). That is, because multiplying $K$ from the left by $L_{e_{i}}^{-1}$ zeros out matrix entries below the $i$-th diagonal block of the product, post-multiplying $L_{e_{i}}^{-1} K$ with $L_{e_{i}}^{-1}$ does not modify the tailing matrix block to the lower right of $i$-th diagonal block of $L_{e_{i}}^{-1} K$. However, as we can see from Line 10 of the pseudo-code in Figure 2, the update of $M$ requires multiplications from both sides because $L_{e_{i}}^{-1} M$ does not zero out matrix entries below the $i$-th diagonal block.

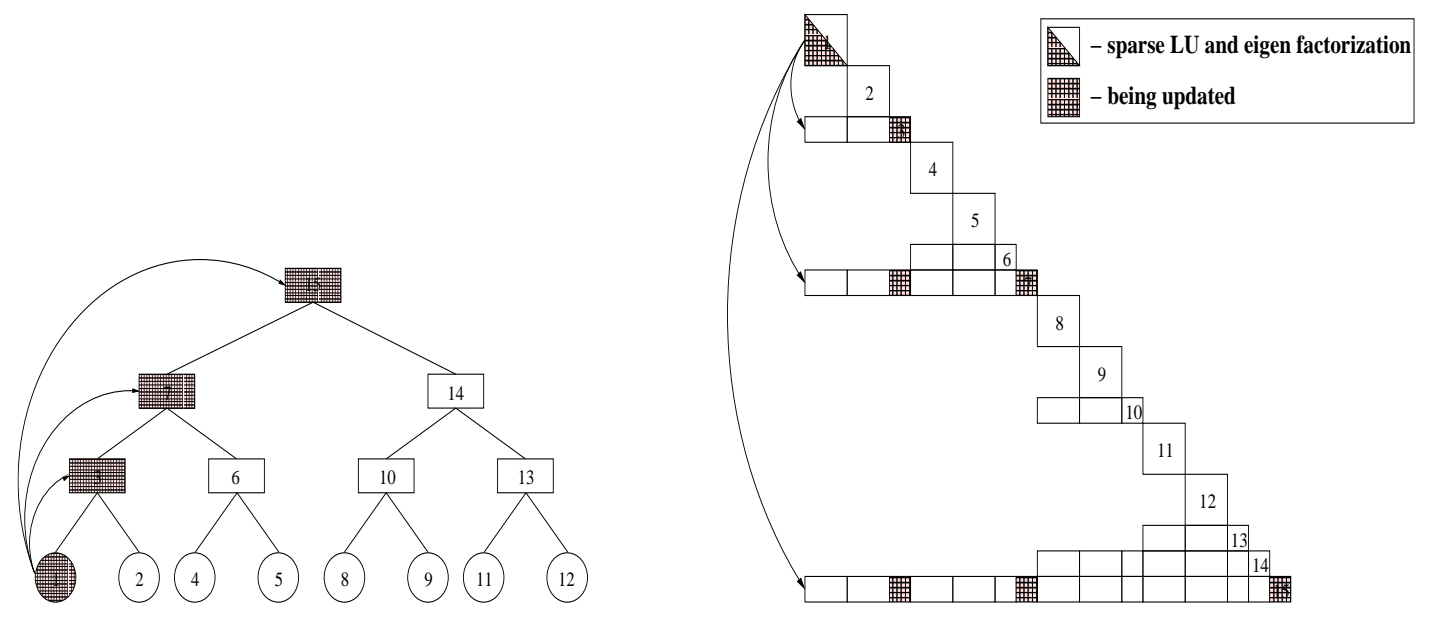

Figure 3: Elimination of leaf node $i=1$ and the associated updates to matrix $K$ and $M$. See lines 8-11 in the code.

Also computed at the leaf node $i$ is a partial eigen-decomposition of the pencil $\left(K_{i i}, M_{i i}\right)$. This calculation is typically done by using a shift-and-invert Lanczos (SIL) procedure. The computed eigenvector matrix $Z_{i}$ is used immediately to partially construct the $i$-th block column of the projected matrix $S^{T} L^{-1} M L^{-T} S$ in Eq. (6). This partial construction is achieved by replacing $M_{j i}$ with $\mathcal{M}_{j i}=\left(M_{j i}-K_{j i} K_{i i}^{-1}\right) Z_{i}$, for $j \in \operatorname{Ancestors}(i)$ (Line 12 of the pseudo-code in Figure 4). 
Since $Z_{i}$ consists of a small subset of the eigenvectors of $\left(K_{i i}, M_{i i}\right), \mathcal{M}_{j i}$ has far fewer columns than $M_{j i}$. As a result, the calculation of $\mathcal{M}_{j i}$ can be completed with a much lower computational complexity.
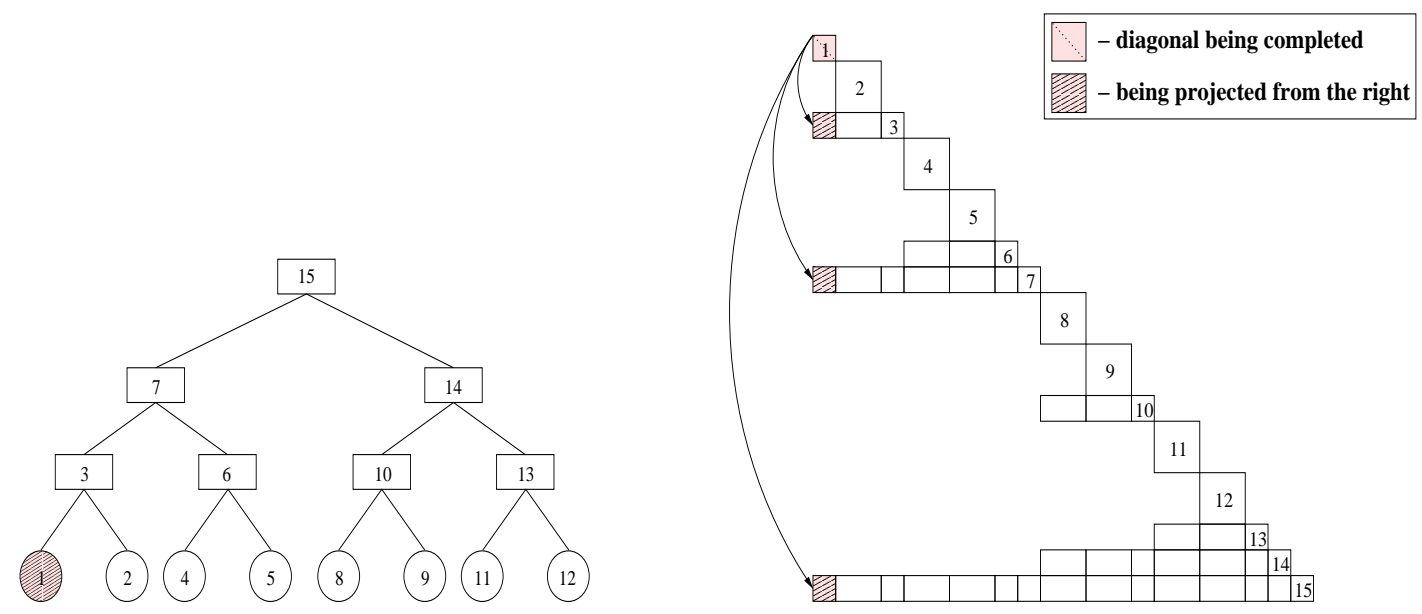

Figure 4: Projection from the right for leaf node $i=1$.

At a higher level tree node $i$, a similar set of operations are performed. What is different at such a node is that the Cholesky factorization of $\widehat{K}_{i i}$ and the partial eigen-decomposition of $\left(\widehat{K}_{i i}, \widehat{M}_{i i}\right)$ (in Lines 16-17 of the pseudo-code in Figure 2) are performed on the diagonal blocks (of $K$ and $M)$ that have been modified by its descendents. These operations cannot be initiated until the contributions from all its descendent nodes have been included.

In addition, the elementary eleminator constructed at such a node is not only applied to block columns of $M$ that are associated with the ancestors of $i$, but also to $\mathcal{M}_{j k}$, where $j \in \operatorname{Ancestors}(i)$ and $k \in$ Desendant $(i)$. This additional step makes the implementation of the AMLS neither a right-looking nor a left-looking algorithm. In Figure 5(a), the dependency of the leaf nodes 1 and 2 on the seperator node 3 is clearly indicated by the direction of the arrows. Figure 5(b) shows that applying the elemintary eliminator constructed at the seperator node 3 to $\mathcal{M}_{j k}$ amounts to subtracting from $\mathcal{M}_{j k}$ a multiple of $\mathcal{M}_{3 k}$ for $j \in \operatorname{Ancestors(3)}=\{7,15\}$ and $k \in$ Desendant(3) = $\{1,2\}$. It is important to recall that $\mathcal{M}_{j k}$ typically has far fewer columns than $M_{j k}$, thus working with $\mathcal{M}_{j k}$ is less costly than working with $M_{j k}$ directly.

Since $\widehat{K}_{i i}$ and $\widehat{M}_{i i}$ are typically dense, and these matrices are much smaller in dimension compared to the diagonal blocks associated with the leaf nodes, the partial eigen-decomposition of $\left(\widehat{K}_{i i}, \widehat{M}_{i i}\right)$ can usually be computed by an LAPACK routine for dense symmetric eigenvalue problems. All eigenvalues and eigenvectors of $\left(\widehat{K}_{i i}, \widehat{M}_{i i}\right)$ are computed, but only a selected number of desired eigenvectors are retained and stored in $Z_{i}$. The matrix $Z_{i}$ is used to partially construct the $i$-th block column of the projected matrix $S^{T} L^{-1} M L^{-T} S$. This partial projection produces $\mathcal{M}_{j i}$ for $j \in \operatorname{Ancestors}(i)$. Moreover, we can now multiply $Z_{i}^{T}$ from the left with $\mathcal{M}_{i k}$ for $k \in$ Descendant $(i)$ to complete the computation of the $i$-th block row of $S^{T} L^{-1} M L^{-T} S$ (shaded blocks in Figure 6 for $i=3$ ). In fact, upon the completion of the matrix operations performed at the seperator node $i$, the leading $i$ block rows and colums of $S^{T} L^{-1} M L^{-T} S$ become available. 

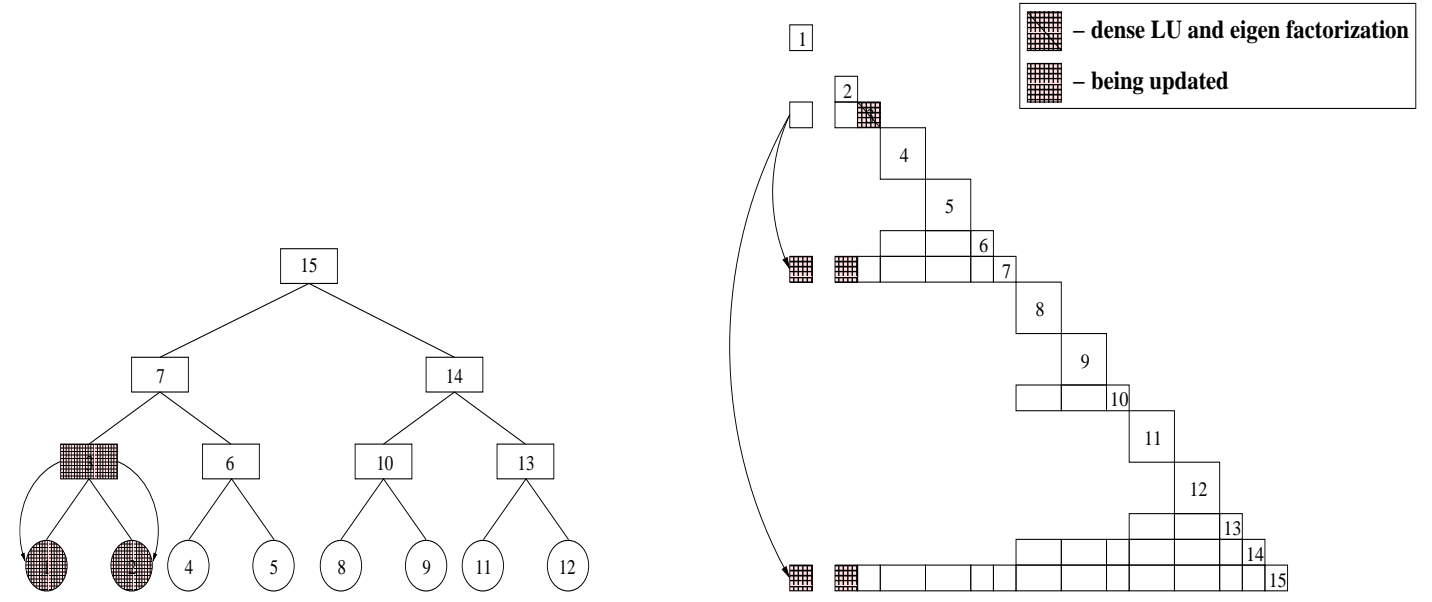

Figure 5: For seperator node $i=3$, more congruence updating is needed for the right-projected blocks in the descendent nodes. See lines 20-22 in the code.

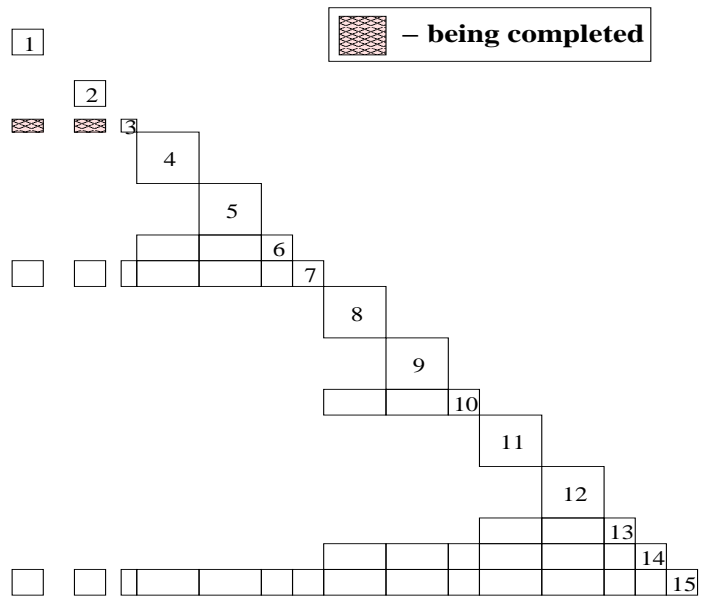

Figure 6: Left-side projection for separator node $i=3$. See lines 30-32 in the code. 


\subsection{A semi-implicit representation of $L$}

When the congruence transformation, the sub-structure mode extraction and the projection calculation (steps (3)-(5) in Figure 1) are interleaved in the way that has been described above, there is no need to store $\widehat{M}=L^{-1} M L^{-T}$. When the $i$-th separator node is being eleminated, the pseudocode listed in Figure 2 maintains $\mathcal{M}_{j i}$ for $i \in \operatorname{Descendant}(i)$. These matrix blocks typically have far fewer columns than $M_{j i}$. Thus the cost for storing them is significantly lower than that for storing the corresponding blocks in $\widehat{M}$. Applying $L_{e_{i}}$ to $M$ will introduce some non-zero fills in $M_{k j}$ for $k, j \in \operatorname{Ancestors}(i)$. However, as we move from the leaf nodes of the separator tree up to the root, the number of these trailing blocks become smaller and smaller. They eventually all vanish as the computation of $S^{T} \widehat{M} S$ is completed. Therefore, by interleaving Steps (2), (3) and (4) of Algorithm 1, we can reduce the amount of memory required in the calculation of $S^{T} \widehat{M} S$.

However, what we have not addressed in Section 3.1 is how $L$ is stored. Due to the non-zero fill introduced during the block Cholesky factorization of $K$, the number of non-zeros in $L$ can potentially be much larger than those in $K$. Hence it is important to develop a technique for reducing memory usage required for $L$.

In the AMLS implementation presented in [9], each block column of $L$ is stored to a disk immediately after it has been used to update $K$ and $M$. Such an out-of-core implementation reduces the memory requirement of AMLS significantly while incurring a modest amount I/O cost.

In this paper, we present an alternative approach that is equally effective. Note that $L$ has the same block structure as $K$ (see (7)), with $L_{j i}=K_{j i} K_{i i}^{-1}$ for a leaf node $i$, and $L_{j i}=\widehat{K}_{j i} \widehat{K}_{i i}^{-1}$ for a separator node $i$. These matrices tend to be denser, thus would require additional storage space to hold the nonzero fill-ins. The basic idea of our approach is to compute and store only the matrix blocks $L_{j i}$ where $i$ is a separator node. All other nonzero matrix blocks in $L$ are not explicitly computed or stored. Whenever $L_{j i}$ is needed, for some leaf node $i$, in the intermediate matrixmatrix multiplication of the form $B=L_{j i} C$, we apply $K_{j i} K_{i i}^{-1}$ to $C$ directly through a sequence of sparse triangular solves and matrix-matrix multiplications instead of forming $L_{j i}=K_{j i} K_{i i}^{-1}$ first. Such a scheme essentially amounts to recomputing $K_{j i} K_{i i}^{-1}$ whenever it is needed. Because a fully explicit representation of $L$ is avoided in our approach, we call this scheme a semi-implicit representation of $L$.

We underlined in Line 10 of the pseudocode in Figure 2 where the recomputation occurs. It is readily seen that the inner $k$-loop would require three matrix-matrix multiplications if $L$ is computed and stored explicitly. In our semi-implicit scheme, the inner $k$-loop requires a triangular solve in addition to three matrix-matrix multiplications. If we assume the cost of a triangular solve is about the same as a matrix-matrix multiplication, the semi-implicit scheme would incur roughly $33 \%$ runtime overhead within the $k$-loop associated with a leaf. However, because constructing the projected pencil $\left(S^{T} \widehat{K} S, S^{T} \widehat{M} S\right.$ ) involves more than just these matrix multiplications, the actual overall runtime increase measured in practice is less than $15 \%$, as we will see in Section 4.2.

To give an intuitive idea of how much space might be saved, let us consider a matrix partition that consists of $\ell$ levels, recomputing the off-diagonal blocks of $L$ instead of storing them explicitly allows us to reduce the total number of stored off-diagonal blocks from $c(\ell)$ to to $c(\ell-1)$, where

$$
c(\omega)=\sum_{j=1}^{\omega} 2^{j} j .
$$

If we assume that each off-diagonal block of $L$ is dense and all off-diagonal blocks are of the same 
size, the percentage of memory saved for $L$ is roughly

$$
\frac{c(l)-c(l-1)}{c(l)}=\frac{2^{l} l}{\sum_{j=1}^{l} 2^{j} j} \geq \frac{2^{l} l}{l \sum_{j=1}^{l} 2^{j}}=\frac{2^{l}}{2\left(2^{l}-1\right)} \approx 50 \% .
$$

But, since computing $S^{T} L^{-1} M L^{-T} S$ without using the semi-implicit scheme would require storing not only $L$ but also selected matrix blocks of $L^{-1} M L^{-T}$, the actual percentage of memory saved is lower than the estimate given here. In Section 4.2, we report the actual memory savings for two specific problems.

\subsection{Computing Ritz pairs}

Since an efficient implmentation of the AMLS algorithm requires one to interleave Steps (2) - (5) outlined in Algorithm 1, we will combine these steps into a single phase of the AMLS implementation. We will later refer to this phase as phase 1 of the AMLS computation. The rest of the AMLS calculation, which involves solving the projected eigenvalue problem (11) and assembling the Ritz vectors $z=L^{-T} S q$, will be lumped into phase 2 of the implementation.

The projected pencil $\left(S^{T} \widehat{K} S, S^{T} \widehat{M} S\right)$ is generally sparse. In fact, $\widetilde{K} \equiv S^{T} \widehat{K} S$ is diagonal, and $\widetilde{M} \equiv S^{T} \widehat{M} S$ typically has the same block nonzero structure as that of $M$. Furthermore, the diagonal blocks of $\widetilde{M}$ are all diagonals. Thus, it is appropriate to use a SIL procedure to compute the desired eigenpairs of $(\widetilde{K}, \widetilde{M})$ in this case. However, when the dimension of the projected problem is relatively small $\left(n_{\text {proj }} \leq 5000\right)$, and when the number of eigenpairs required is a large fraction of $n_{\text {proj }}$, we observe that it is often more efficient to use a dense eigensolver to compute all eigenpairs of $(\widetilde{K}, \widetilde{M})$.

When $n_{\text {proj }}$ becomes very large, SIL can be computationally costly too. To reduce the cost of the Ritz value calculation, Kaplan proposed an alternative, special subspace iteration (SSI) scheme [9]. Kaplan's scheme first reduces the projected pencil to an even smaller pencil by removing rows and columns of $(\widetilde{K}, \widetilde{M})$ associated with the "undesirable" diagonal entries in $\widetilde{K}$. Eigenpairs of this reduced pencil $\left(\widetilde{K}_{B}, \widetilde{M}_{B}\right)$ are computed by the SIL algorithm. The $n_{k}$ Ritz vectors obtained from SIL are used as the starting basis for a block inverse iteration, where $n_{k}$ is larger than the number of eigenvalues wanted. Denote this starting subspace by $Y^{(0)}$. The $j$ th iteration of the SSI scheme comprises the following steps. First, one step of inverse iteration of the form $X^{(j)}=\widetilde{K}_{B}^{-1} \widetilde{M}_{B} Y^{(j-1)}$ produces another subspace $X^{(j)}$ of size $n_{k}$. Second, each vector from $X^{(j)}$ is paired with the corresponding Ritz vector in $Y^{(j-1)}$ to form a two-dimensional subspace, from which the best approximation to the lowest eigenpair is computed. Third, the $n_{k}$ Ritz vectors obtained from the two-dimensional projected problems form a new subspace to project the pencil $\left(\widetilde{K}_{B}, \widetilde{M}_{B}\right)$, and the $n_{k}$ approximate eigenvectors computed from this projection spans the subspace $Y^{(j)}$ for the next iterate. The cost of the Kaplan scheme is proportional to the number of block inverse iterations performed. Performing more inverse iterations improves the accuracy of the approximate eigenpairs but increases the runtime. We will compare the cost and accuracy of the Kaplan's scheme with those of SIL in the next secition.

\section{Performance Evaluation}

Since the AMLS algorithm involves only algebraic operations, its implementation does not depend on application specific knowledge. However, the performance of the algorithm may vary from one problem to another due to differences in the sparsity structure and spectral properties of the 
problems. Up until now, the performance of the algorithm has only been evaluated and analyzed for problems that arise from structural engineering [9, 12]. It has been shown that the performance of AMLS compares favorably with the Lanczos algorithm and the algorithm can reduce the frequency repsonse calculation time by several orders of magnitude on workstations [9].

In this section, we examine the general performance of AMLS by applying it to eigenvalue problems arising from two other types of applications.

In the first problem, the stiffness and mass matrices are obtained from a finite element discretization of a six-cell damped detuned accelerator structure (DDS) [11]. The eigenvalues of interest are related to the cavity resonance frequencies of the accelerator and the corresponding eigenvectors are used to model the electromagnetic accelerating field.

The second problem we include here arises from the normal mode vibrational analysis of the polyethylene (PE) particles [17]. In this application, we are interested in the low frequency vibrational modes of the PE molecule, which can be solved by computing the eigenvalues and eigenvectors associated with the Hessian of a potential function that describes the interaction between different atoms. The dimension of the Hessian matrix is three times the number of atoms in the molecule.

Table 1 summarizes the dimension and sparsity of the test cases. Figure 7 shows the geometric model of the six-cell accelerator cavity structure and the sparsity pattern of the matrix $|K|+|M|$ after $K$ and $M$ are permuted by Metis. Figure 8 shows the molecular structure of the PE3K particle and the sparsity pattern of the Hessian matrix after it is permuted by METis.

\begin{tabular}{|l|r|r|l|}
\hline Problem & Dimension & $\mathrm{nnz}(\mathrm{K}+\mathrm{M})$ & Discipline \\
\hline DDS6_COARSE & 5584 & 95138 & accelerator, 6 cells, coarse grid \\
DDS6_FINE & 65740 & 1455772 & accelerator, 6 cells, fine grid \\
PE3K & 9000 & 3279690 & vibrational analysis, 3000 atoms \\
PE12K & 36000 & 7128473 & vibrational analysis, 12000 atoms \\
\hline
\end{tabular}

Table 1: Characteristics of the test problems.
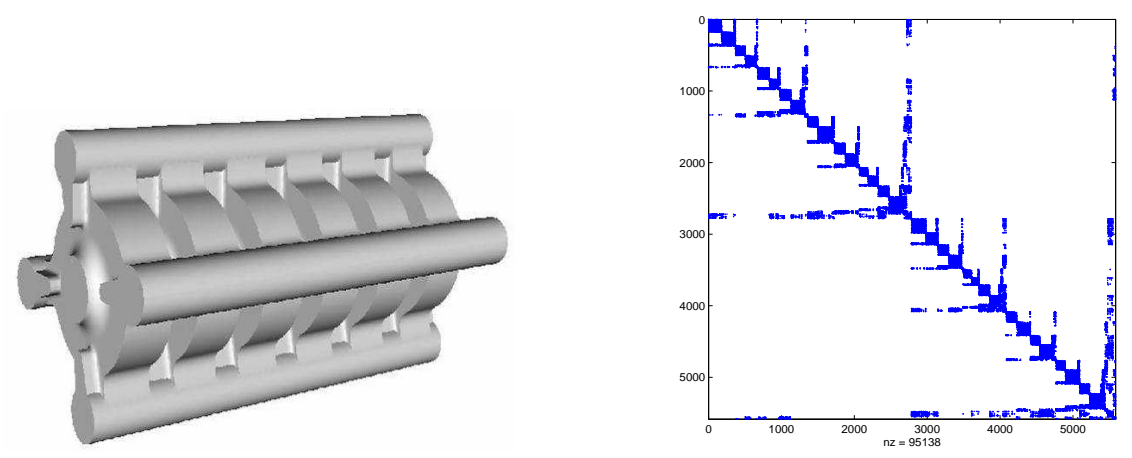

Figure 7: The accelerator cavity of DDS6_COARSE and the sparsity pattern of $|K|+|M|$ after it is permuted by Metis.

As we will see in the following, the performance of AMLS, which can be measured by runtime, memory requirement and the accuracy of the computed eigenvalues, depends on a number of choices one has to make at runtime. These choices include, for example, the number of partitioning levels $\left(n_{\text {levels }}\right)$, the number of eigenpairs $\left(n_{\text {modes }}\right)$ one must compute and choose from each sub-structure, and the method one uses to compute the eigenpairs of the final projected problem.

Although it remains difficult to identify the optimal choices for some of the run time parameters 

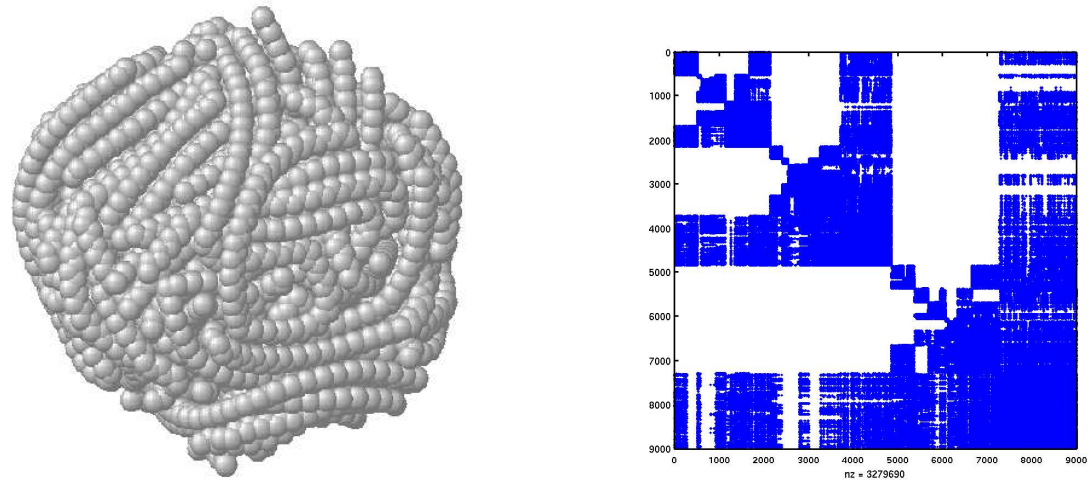

Figure 8: The molecular structure of PE3K and the sparsity pattern of the Hessian after it is permuted by MeTis.

a priori, the following performance evaluation demonstrates the effect of these parameters on the performance of AMLS and the trade-offs between different choices. In addition, the empirical results presented below provide some general guidelines on how to change these paramters in order to achieve better performance.

All computational experiments reported below are carried out on a single IBM Power3 processor with a clock speed of $375 \mathrm{Mhz}$ and $2 \mathrm{MB}$ of level-2 cache. The external software packages used are: Metis [10], ARPACK [13], SuperLDLT [14] and SuperLU [5]. We compiled all the codes using IBM's xlc (C) or xlf (Fortran) compilers with -03 optimization flag. We use nev to denote the number of wanted eigenvalues, $n_{\text {levels }}$ to denote the number of partitioning levels, and $n_{\text {modes }}$ to denote the number of modes chosen from each sub-structure. The accuracy tolerance for each subproblem is denoted by $\tau_{s u b}$, and the accuracy tolerance for the projected problem is denoted by $\tau_{\text {proj. }}$. In all the experiments, we set $\tau_{\text {sub }}=10^{-10}$ and $\tau_{\text {proj }}=10^{-5}$.

\subsection{Variation of runtime with respect to the number of sub-structures}

In the first set of experiments, we try to assess the variation in the overall runtime of AMLS with respect to the number of partitioning levels $\left(n_{\text {levels }}\right)$. In general, as one increases the number of levels in algebraic partitioning, the number of spectral components that can be chosen from each sub-structure is reduced. The exact number of spectral components that should be chosen from each sub-structure is problem dependent, and the selection rule should be based on the theory and heuristics established in [16]. However, to focus on the effect of partitioning depth, we set aside the accuracy issue here and keep the total number of modes selected from all sub-structures roughly the same for all partitioning schemes. That is, if we double the number of sub-structures by dividing each sub-structuring into two smaller sub-structures, we then reduce $n_{\text {modes }}$ associated with each sub-structure by a half.

Table 2 shows the timing statistics for the DDS6_Fine problem. Since the separators in this problem are small, all the coupling modes are retained in the subspace (10). In addition to the total time, we examined the timing breakdown of the two major phases of the implementation defined in Section 3.3. Recall that in the first phase of the implementation, Steps (2)-(5) of Algorithm 1 are interleaved. In the second phase, we compute the Ritz approximation to the desired eigenpairs. The cost of the first phase depends on $n_{\text {levels }}$ and the number of modes selected from the sub-structures $\left(n_{\text {modes }}\right)$. The cost of the second phase depends on the total number of eigenpairs of $(K, M)$ to be computed $(n e v)$ in addition to $n_{\text {levels }}$ and $n_{\text {modes }}$. 
The total amount of time required to compute the smallest 100 eigenpairs of this problem is listed in Column 5 of the table for different choices of $n_{\text {levels }}$. We observe that the best timing is obtained when $n_{\text {levels }}=3$. As a comparison, computing these eigenpairs by a shift-and-invert Lanczos (SIL) method (using ARPACK and SuperLLT packages [14] with METIS reordering) would take 407 seconds. Thus when nev $=100$, AMLS with $n_{\text {levels }}=3$ is competitiveve with SIL in speed. In Columns 3 and 4 of the table, we show how the runtime is split between the first and the second phases of the AMLS calculation. We observe that the phase 1 calculation becomes less time consuming as we increase $n_{\text {levels }}$. However, increasing $n_{\text {levels }}$ leads to a rapid increase in the runtime spent in the second phase of the AMLS calculation. Such an increase is caused by the increased dimension of the projected problem, which is in turn caused by an exponential increase of the number of separators with respect to $n_{\text {levels }}$. We observe that the time spent in the second phase of the AMLS calculation actually exceeds that spent in the first phase for the DDS6_FINE problem when $n_{\text {levels }}>3$.

\begin{tabular}{|c|c||ccc|}
\hline$n_{\text {levels }}$ & $n_{\text {modes }}$ & $\begin{array}{c}\text { phase 1 } \\
(\mathrm{sec})\end{array}$ & $\begin{array}{c}\text { phase 2 } \\
(\mathrm{sec})\end{array}$ & $\begin{array}{c}\text { total } \\
(\mathrm{sec})\end{array}$ \\
\hline \hline 2 & 100 & 458 & 33 & 491 \\
\hline 3 & 50 & 288 & 77 & 365 \\
\hline 4 & 25 & 220 & 235 & 455 \\
\hline 5 & 12 & 194 & 292 & 486 \\
\hline 6 & 6 & 152 & 352 & 504 \\
\hline
\end{tabular}

Table 2: Runtime with different $n_{\text {levels }}$. Problem DDS6_FINE, nev $=100$.

The timing statistics for the PE3K problem shows a similar pattern to that reported in Table 2 . In this problem, we compute 500 smallest eigenpairs. The separators produced by the METIS nested dissection reordering have relatively large dimensions. We chose roughly $n_{\text {sep }} / 5$ coupling modes from each separator, where $n_{s e p}$ is the size of the separator. As we can see in Table 3 , the CPU time spent in phase 1 of AMLS also decreases as $n_{\text {levels }}$ increases. The increase in phase 2 time is less dramatic than that of the DDS6_FINE problem, because the size of the final projected problem increases slowly for PE3K. The best overall performance is obtained when $n_{\text {levels }}=6$. In this case, AMLS is almost twice as fast as SIL which used 956 seconds to compute all desired eigenpairs.

\begin{tabular}{|c|c||ccc|}
\hline$n_{\text {levels }}$ & $n_{\text {modes }}$ & $\begin{array}{c}\text { phase 1 } \\
(\mathrm{sec})\end{array}$ & $\begin{array}{c}\text { phase 2 } \\
(\mathrm{sec})\end{array}$ & $\begin{array}{c}\text { total } \\
(\mathrm{sec})\end{array}$ \\
\hline \hline 2 & 100 & 462 & 55 & 521 \\
\hline 3 & 50 & 439 & 81 & 523 \\
\hline 4 & 25 & 404 & 98 & 505 \\
\hline 5 & 12 & 391 & 105 & 499 \\
\hline 6 & 6 & 381 & 98 & 483 \\
\hline
\end{tabular}

Table 3: Runtime with different $n_{\text {levels }}$. Problem PE3K, nev $=500$. 


\subsection{Variation of memory usage with respect to the number of sub-structures}

We now assess the variation in AMLS memory usage with respect to $n_{\text {levels }}$. Here, our experimental setup is the same as that in Section 4.1. Table 4 shows the memory usuage statistics for the DDS6_FINE problem. Column 3 gives the total memory usage for different choices of $n_{\text {levels }}$ when a semi-implicit representation of $L$ is activated. The actual amount of savings gained from this semi-implicit scheme is listed in column 4. In Column 5, we list the estimated the percentage of savings based on the formula $\frac{c(l)-c(l-1)}{c(l)}$ given in Section 3.2, where $c(\ell)$ is defined in Eqn. (19).

As we explained in Section 3, a semi-implicit representation of $L$ would incur a slight increase in runtime because the off-diagonal blocks of $L$ that are not stored must be recomputed when they are needed. The extra cost associated with recomputing is reported in Column 6 . We see that the actual recomputing overhead reported in Table 4 is lower than the rough estimation made in Section 3 (about 33\%). This is because our estimation does not account for the time spent in the other part of the algorithm. We observe that up to $50 \%$ of the memory usage can be reduced in AMLS for the DDS6_FINE problem with only 10-15\% extra runtime. This reduction is very attractive when memory is at a premium.

\begin{tabular}{|c|c||cccc|}
\hline$n_{\text {levels }}$ & $n_{\text {modes }}$ & $\begin{array}{c}\text { total-mem } \\
(\mathrm{MB})\end{array}$ & $\begin{array}{c}\text { mem-saved } \\
(\mathrm{MB})\end{array}$ & $\begin{array}{c}\text { mem-saving } \\
\text { estimate }\end{array}$ & $\begin{array}{c}\text { recompute } \\
\text { time (sec) }\end{array}$ \\
\hline \hline 2 & 100 & 319 & $199(38.4 \%)$ & $75.0 \%$ & $9.2(1.5 \%)$ \\
\hline 3 & 50 & 263 & $263(50.0 \%)$ & $66.7 \%$ & $51.5(11.0 \%)$ \\
\hline 4 & 25 & 325 & $248(43.3 \%)$ & $62.5 \%$ & $60.7(13.3 \%)$ \\
\hline 5 & 12 & 392 & $228(36.8 \%)$ & $60.0 \%$ & $64.0(13.2 \%)$ \\
\hline 6 & 6 & 480 & $192(28.6 \%)$ & $58.3 \%$ & $55.3(10.9 \%)$ \\
\hline
\end{tabular}

Table 4: Memory usage with different $n_{\text {levels }}$. Problem DDS6_FINE, nev $=100$.

As a comparison, the memory usage measured in SIL is 308 Megabytes(MB). Thus, our implementation of AMLS appears to be more efficient in both runtime and memory usage than SIL when $n_{\text {levels }}$ is set to 3 .

For the PE3K problem, the percentage of memory saved using the semi-implicit representation of $L$ is not as impressive as that reported for the DDS6_FINE problem. The reason we observed a smaller amount of saving is that this problem has relatively large separators. Since the off-diagonal blocks of $L$ associated with each separator are kept in memory, the amount of memory that can be saved is limited. However, even in this case, we observe from Table 5 that up to $28 \%$ memory is saved. The optimal memory usage $(265 \mathrm{MB})$ is achieved when $n_{\text {levels }}=6$. This compares favorably with the use of $283 \mathrm{MB}$ memory in the SIL calculation.

\begin{tabular}{|c|c||cccc|}
\hline$n_{\text {levels }}$ & $n_{\text {modes }}$ & $\begin{array}{c}\text { total-mem } \\
(\mathrm{MB})\end{array}$ & $\begin{array}{c}\text { mem-saved } \\
(\mathrm{MB})\end{array}$ & $\begin{array}{c}\text { mem-saving } \\
\text { estimate }\end{array}$ & $\begin{array}{c}\text { recompute } \\
\text { time (sec) }\end{array}$ \\
\hline \hline 2 & 100 & 289 & $113(28.1 \%)$ & $75.0 \%$ & $73.9(14.2 \%)$ \\
\hline 3 & 50 & 271 & $96(26.2 \%)$ & $66.7 \%$ & $82.6(15.8 \%)$ \\
\hline 4 & 25 & 268 & $66(19.8 \%)$ & $62.5 \%$ & $57.4(11.4 \%)$ \\
\hline 5 & 12 & 270 & $42(13.5 \%)$ & $60.0 \%$ & $35.9(7.2 \%)$ \\
\hline 6 & 6 & 265 & $34(11.4 \%)$ & $58.3 \%$ & $29.6(6.1 \%)$ \\
\hline
\end{tabular}

Table 5: Memory usage with different $n_{\text {levels }}$. Problem PE3K, nev $=500$. 


\subsection{Solving the projected problem}

Table 2 shows that solving the projected eigenvalue problem (6) can become more expensive than the computation required to construct the projected pencil $\left(S^{T} \widehat{K} S, S^{T} \widehat{M} S\right)$ when the number of partitions is relatively large. Even if we reduce the number of spectral components selected from each sub-structure (by as much as a half) as we did for DDS6_FINE in Table 2, the dimension of the projected problem, $n_{\text {proj }}$, is still likely to increase as the number of partitions increases. Such an increase in dimension is caused by the increased number of interface blocks (seperators) produced by the multi-level matrix partitioning. Thus, to reduce the total computational cost of AMLS, one must choose an effcient algorithm to compute the desired eigenpairs of the projected pencil.

Table 6 shows the changes in $n_{\text {proj }}$ and the CPU time required to compute the smallest nev $=$ 500 eigenpairs of $(\widetilde{K}, \widetilde{M})$ obtained by applying AMLS to the DDS6_FINE problem with different choices of $n_{\text {levels }}$. We list the CPU time required by both the SIL and the LAPACK divideand-conquer eigenvalue calculation routine DSYEVD. For this example, $n_{\text {modes }}$ is set to 100 when $n_{\text {levels }}=2$, and is reduced by a half when $n_{\text {levels }}$ is increased by one. All spectral modes of the interface blocks are selected. Thus, the increase in $n_{\text {proj }}$ reflects the increased number of seperators produced by nested dissection. We observe that when $n_{\text {proj }}<5000$, DSYEVD is significantly faster than SIL, and SIL is only slightly faster when $n_{\text {proj }}$ reaches 6547 .

\begin{tabular}{|c|c|c|r|r|}
\hline$n_{\text {levels }}$ & $n_{\text {modes }}$ & $n_{\text {proj }}$ & SIL (sec) & DSYEVD (sec) \\
\hline \hline 2 & 100 & 1289 & 557 & 33 \\
\hline 3 & 50 & 1878 & 744 & 77 \\
\hline 4 & 25 & 2976 & 986 & 235 \\
\hline 5 & 12 & 4432 & 1206 & 651 \\
\hline 6 & 6 & 6547 & 2036 & 2064 \\
\hline
\end{tabular}

Table 6: Solving the final projected eigenvalue problem for DDS6_FINE. $n e v=500$.

We also experimented with Kaplan's special subspace iteration (SSI) scheme discussed in Section 3.3 using the DDS6_COARSE problem with $n_{\text {levels }}=1$. The dimensions of the two sub-structures produced by this one-level partition are 2761 and 2791 respectively. The size of seperator in this case is 32 . We set $n_{\text {modes }}$ to 796 and 802 on each sub-structure respectively. All coupling modes are included in $S$. In Figure 9, we plot the relative accuracy of the 50 Ritz values computed by both SIL and SSI. We experimented with performing different numbers of block inverse iterations in SSI, and will denote an SSI run with $k$ block inverse iterations by SSI(k). Figure 9 shows that it takes at least three inverse iterations in SSI to produce Ritz values that are as accurate as those produced by SIL. The CPU time required to perform three block inverse iterations in SSI is comparable to that required to carry out SIL on $(\widetilde{K}, \widetilde{M})$. Therefore, we do not see a clear advantage of using Kaplan's scheme over using SIL for this problem.

\subsection{Variation of runtime with respect to nev}

Since the computational complexity of AMLS is slightly higher than that associated with a block $L D L^{T}$ factorization of $K$, one should not expect a significant performance gain from AMLS over SIL when the number of eigenvalues to be computed (nev) is small (say less than 100). In fact, Table 2 shows that in the case of DDS6_FInE, the performance of AMLS is slightly worse than SIL when $n e v=100$ and $n_{\text {levels }}=2,4,5$ and 6 . 


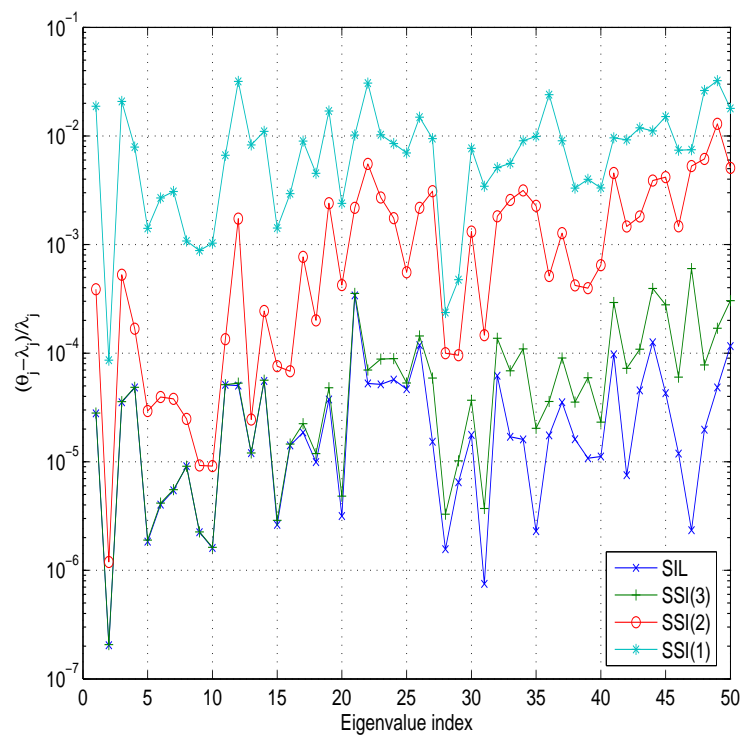

Figure 9: Accuracy of Kaplan's subspace inverse iteration scheme (SSI) for the projected eigenvalue problem. Problem DDS6_COARSE, nev $=50$.

However, as nev increases, AMLS becomes more attractive as we can see from Figure 10 where we plotted both the AMLS and SIL runtime as a function of nev. If we assume that a sufficient number of spectral modes have already been selected from each sub-structure so that the projected pencil $(\widetilde{K}, \widetilde{M}) \equiv\left(S^{T} L^{-1} K L^{-T} S, S^{T} L^{-1} M L^{-T} S\right)$ contains accurate approximations to a large number of eigenpairs of $(K, M)$, an increase in nev simply means that we need to compute more Ritz pairs from $(\widetilde{K}, \widetilde{M})$. In this case, the incremental cost of AMLS associated with computing more approximate eigenpairs can be completely accounted for by the additional computation required to solve the projected eigenvalue problem. When the dimension of the $(\widetilde{K}, \widetilde{M})$ is much smaller than $n$, such an incremental cost is relatively small compared to the cost of constructing $(\widetilde{K}, \widetilde{M})$.

In contrast, the incremental cost of SIL can be much higher than the cost of computing the initial block $L D L^{T}$ factorization. If a single $L D L^{T}$ factorization is used in SIL, the convergence of eigenvalues far away from the target shift can be very slow, leading to a significant increase in the size of the Lanczos basis required to capture all the desired spectral components of $(K, M)$ or a increase in the number of restarts [13] required to filter out the unwanted spectral components from the Krylov subspace constructed by SIL. In either case, the number of sparse triangular substitutions would increase substantially and the cost of maintaining the orthonormality of the Lanczos basis would become higher also. The use of multiple target shifts in SIL has the benefit of accelerating the convergence of the desired eigenpairs, and thus reduces the overall number of triangular substititions. However, because the $L D L^{T}$ factorization of $K-\sigma M$ must be recomputed for each target shift $\sigma$, the cost of SIL typically increases linearly with respect to the number of shifts used. In Figure 10, the estimated runtime of SIL is based on the assumption that a single shift is used to compute at most 100 eigenpairs of $(K, M)$, i.e., when $n e v=500$, five SIL runs with five different target shifts are used to compute all desired eigenvalues. It is easy to see from this figure that AMLS is clearly a more favorable choice than SIL for the DDS6_FINE problem when $n e v>200$. 


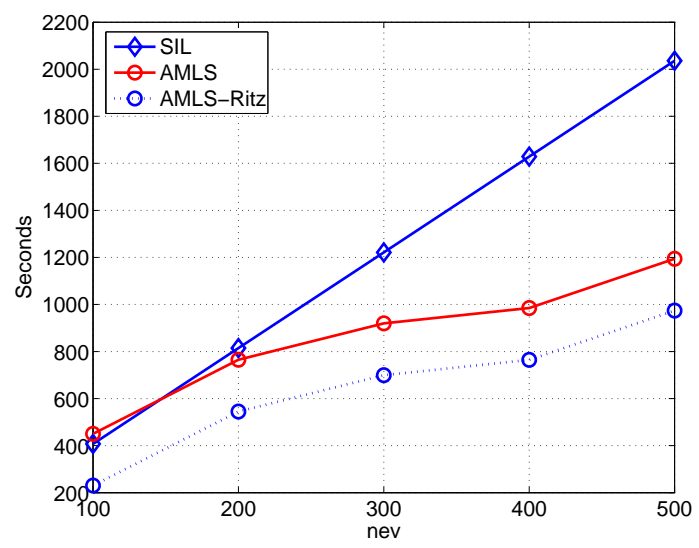

Figure 10: Runtime of AMLS and SIL with increasing nev. Problem DDS6_FINE, $n_{\text {levels }}=4$, $n_{\text {modes }}=25$.

\subsection{Accuracy}

The accuracy of AMLS clearly depends on the number of spectral modes $\left(n_{\text {modes }}\right)$ selected from each sub-structure. For a fixed sub-structure partition, increasing $n_{\text {modes }}$ increases the dimension of the subspace $\mathcal{S} \equiv \operatorname{span}\{S\}$ onto which $(K, M)$ is projected. Such an increase naturally results in improved accuracy in the Rayleigh-Ritz approximations to the desired eigenpairs. However, the amount of improvement varies from one problem to another. Figure 11(b) shows that, for problem DDS6_FINE, by increasing $n_{\text {modes }}$ on all sub-structures of a 5-level partition uniformly from 12 to 100 , one improves the accuracy of the leading 100 Ritz values by roughly one digit. Such a modest improvement for this problem can be explained by the slow decay of the $\rho$-factor (see Eqn. (18)) as a function of eigenvalues of each sub-structure. Since the $\rho$-factor provides an estimation of how significant the corresponding sub-structure mode is to the approximate eigenvector [16], a slowly decreasing $\rho$-factor implies that a small change in $n_{\text {modes }}$ results in limited change of accuracy in the approximate eigenvector.

A mode selection scheme based on an appropriate $\rho$-factor cutoff would guarantee sufficient accuracy of the Rayleigh-Ritz approximation produced by AMLS [6, 16]. However, it has been observed that such a selection scheme often retains more modes from each sub-structure than is necessary. It is shown in [16] that the contribution of each mode to the approximate eigenvector should be measured by the magnitude of the corresponding element in the $y_{i}$ vector defined in (14). There are cases in which elements of $y_{i}$ are tiny (indicating these modes can be discarded) while the corresponding $\rho$-factors are relatively large. Unfortunately, $y_{i}$ cannot be computed in advance. Thus, further research is needed to develop additional heuristics for estimating the magnitude of each element of $y_{i}$ more accurately.

The issue of mode selection verus accuracy becomes more complex when one takes into account the different choices of sub-structure partitions. As $n_{\text {levels }}$ increases, each sub-structure contains fewer modes to choose from, thus one would naturally decrease $n_{\text {modes }}$ on each sub-structure. Ideally, one would like to decrease $n_{\text {modes }}$ such that the dimension of $\mathcal{S}$ (which is the sum of all $n_{\text {modes }}$ associated different sub-structures and the interface blocks) remain roughly the same. However, Figure 11 shows that such a strategy typically leads to a loss of accuracy in the Ritz values extracted from $\mathcal{S}$. Thus, one may be forced to choose a relatively large $n_{\text {modes }}$ on each sub-structure, which would increase the dimension of $\mathcal{S}$. Although increasing the number of sub- 
structures typically reduces the cost of constructing $S^{T} L^{-1} M L^{-T} S$, an increase in the dimension of $\mathcal{S}$ makes solving the projected problem more costly. Further research is required to identify the optimal balance.



(a) Increasing levels

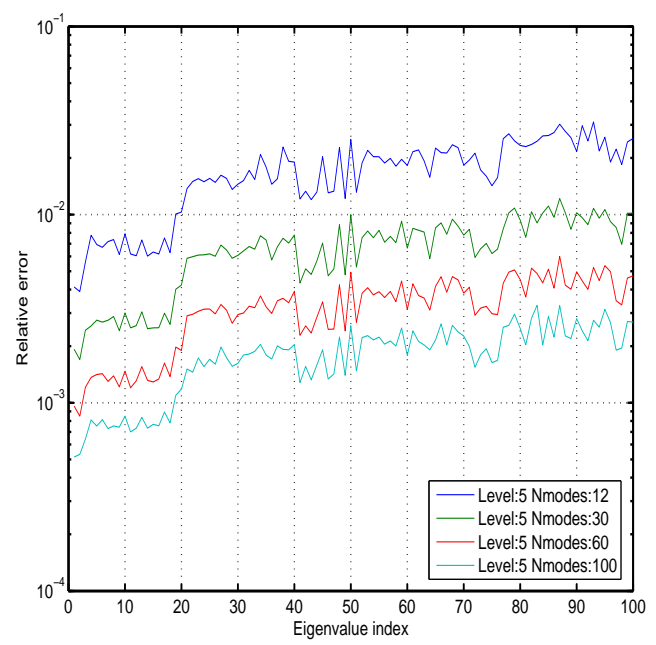

(b) Fixed level, increasing $n_{\text {modes }}$

Figure 11: Eigenvalue accuracy of DDS6_FINE.

\subsection{Partial mode selection for the interface blocks}

For many 3D problems, a multi-level partitioning of $|K|+|M|$ produces relatively large-sized seperators. For these problems, retaining all the spectral components of each seperator in the the subspace represented by (5) or (10), as proposed in the CMS method, would result in a large projected pencil. Even when the size of each seperator is modest, the exponential increase of the number of seperators with respect to $n_{\text {levels }}$ can make solving the projected problem (6) a computationally intensive task. Thus, in many cases, it is desirable to reduce the number of spectral modes chosen from each seperator so that the dimension of the projected pencil can be reduced.

In [9], the number of modes selected from each seperator is determined by a cutoff frequency set slightly larger than the highest frequency of interest for the frequency response. For applications that do not arise from frequency reponse analysis, such a cutoff typically does not exist. Even when such a cutoff frequency exists, the error analysis presented in Section 2.3 indicates that using a given cutoff frequency may not lead to an optimal mode selection strategy.

In this section, we illustrate the effect of separator partial mode selection on the accuracy and performance of the AMLS computation with two examples.

In the first example, we take a close look at the effect of partial seperator mode selection on the approximation to the smallest eigenvalues of the DDS6_COARSE problem. In this example, a single-level partitioning is performed to obtain two sub-structures. They are connected by a separator of size 34 . We set $n_{\text {modes }}$ associated with the first and second sub-structures to 109 and 108 respectively, and vary the number of modes selected from the seperator. Figure 12 shows that reducing $n_{\text {modes }}$ from 34 to 9 on the separator has a large effect on the accuracy of the approximation to some eigenvalues but not others. For example, the relative error of the approximation to $\lambda_{1}$ 
remains the same regardless how $n_{\text {modes }}$ is chosen for the separator. However, the relative error of the approximation to $\lambda_{2}$ increases by almost three orders of magnitude as we decrease $n_{\text {modes }}$ from 29 to 9.

To understand what causes such difference, we examine the magnitude of the elements of $y_{i}(i=$ $1,2,3)$ associated with $\lambda_{1}$ and $\lambda_{2}$. The theory we established in [16] and summarized in Section 2.3 suggests that the accuracy of the approximate eigenvalues are determined by the magnitude of the truncated elements in $y_{i}$. We represent these elements by a vector $h_{i}$ defined by

$$
h_{i}=\left(\begin{array}{ll}
0 & I_{k_{i}}
\end{array}\right) y_{i}
$$

where $k_{1}=109$ and $k_{2}=108$.

In the top row of Figure 12(b), we plot the magnitude of the vectors $h_{1}, h_{2}$ and $y_{3}$ associated with $\lambda_{1}$. We observe that the magnitude of most elements in $y_{3}$ is comparable to or less than that of $h_{1}$ and $h_{2}$. Thus truncating these elements by excluding the corresponding coupling modes from $S_{3}$ has very little effect on the accuracy of the approximation to $\lambda_{1}$.

In the bottom row of Figure 12(b), we plot the magnitude of the vectors $h_{1}, h_{2}$ and $y_{3}$ associated with $\lambda_{2}$. We notice that the magnitude of many elements in $y_{3}$ are considerably larger than the largest element in $h_{1}$ or $h_{2}$. Thus the accuracy of the approximation to $\lambda_{2}$ depends largely on whether the coupling modes corresponding to these elements are included in $S_{3}$.

In the second application, we illustrate that performing partial mode selection can have a significant impact on the performance of AMLS on the PE3K and PE12K problems. Both of these matrice contain separators of large dimensions. We set $n_{\text {modes }}$ to 100 for each sub-structure. With a three-level partitioning, the cumulative size of the seperator in PE3K is roughly one-fourth of dimension of the original problem. For PE12K, the size of the top level seperator is already 4937. Table 7 shows the reduction in dimension of the projected pencil $(\widetilde{K}, \widetilde{M})$ when roughly $20 \%$ of the modes are selected from each seperator for both PE3K and PE12K. Such a reduction translates into a reduction of the AMLS runtime by at least a half for both PE3K and PE12K as shown in Table 8. Table 8 also shows that with partial selection, AMLS runtime compares favorably with that of SIL.

\begin{tabular}{|c|c|c|c|}
\hline Problem & $n_{\text {levels }}$ & partial & full \\
\hline \hline PE3K & 3 & 1484 & 5658 \\
\hline PE12K & 4 & 5350 & 20348 \\
\hline
\end{tabular}

Table 7: Dimension of the projected problem with and without partial selection.

\begin{tabular}{|c|c|c|c|c|c|}
\hline Problem & nev & $n_{\text {levels }}$ & partial & full & SIL \\
\hline \hline PE3K & 500 & 3 & 581 & 1776 & 1061 \\
\hline PE12K & 1000 & 4 & 15511 & $>36000$ & 18000 \\
\hline
\end{tabular}

Table 8: Effect of partial mode selection on the AMLS runtime (in seconds).

In our experiment, the number of modes selected from each seperator is determined by examining the magnitude of the $\rho_{\lambda_{1}}$ factor associated with the top-level seperator. Figure 13 shows that such a selection heuristic allows us to maintain at least two digits of accuracy in the approximation to the smallest eigenvalue of both PE3K and PE12K. 


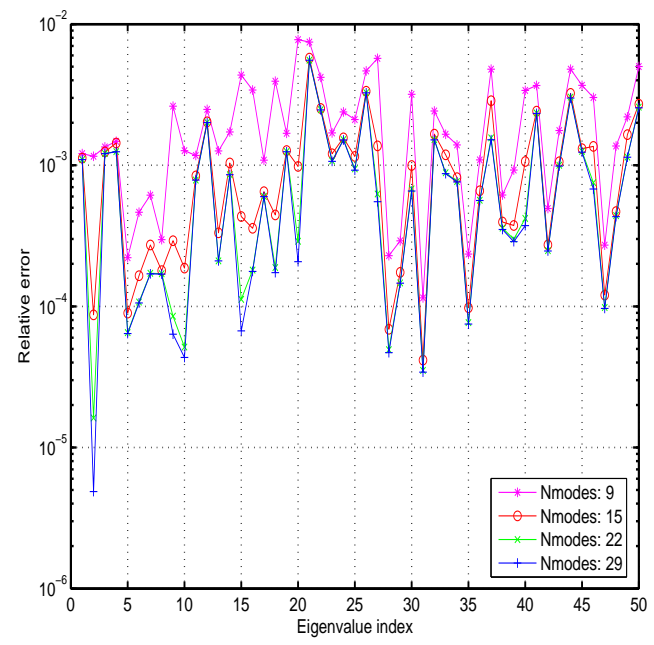

(a) Decreasing $n_{\text {modes }}$ for the separator


(b) $h_{1}, h_{2}$ and $y_{3}$ for the first and the second eigenvectors

Figure 12: Partial mode selection for the coupling modes. Problem DDS6_COARSE, one level partitioning is used. $n e v=50$. 


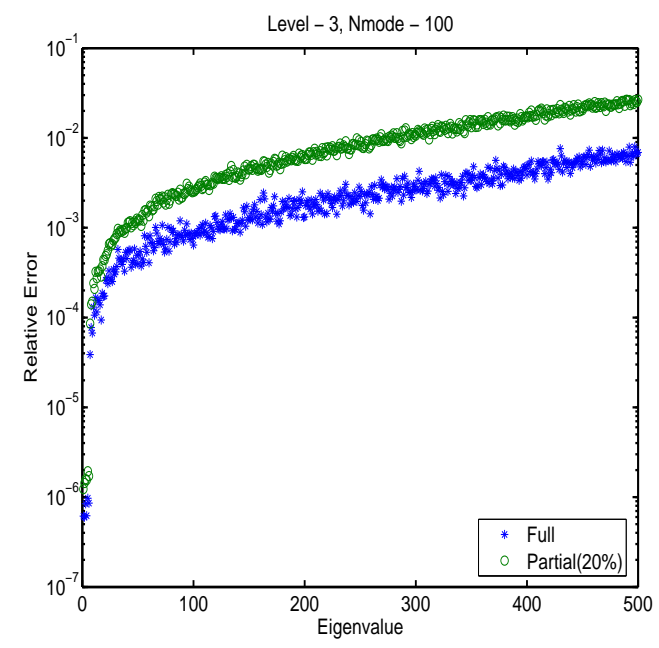

(a) Relative error of the approximation to the smallest 500 eigenvalues of PE3K

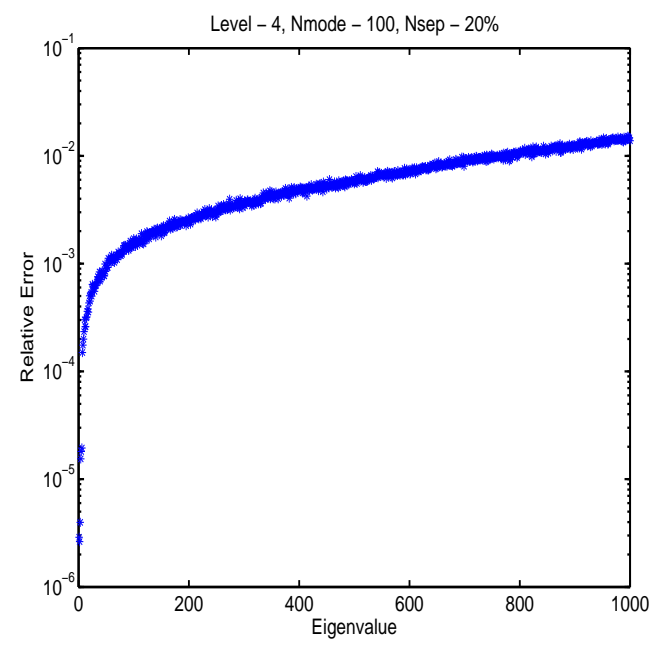

(b) Relative error of the approximation to the smallest 1000 eigenvalues of PE12K

Figure 13: Relative error

\section{Concluding Remarks}

An efficient implementation of the AMLS algorithm requires matrix operations performed in the congruence transformation of $(K, M)$, the sub-structure eigenpair calculation, and the projection of $(K, M)$ to be organized in a way that would lead to a minimal floating point operation count and memory usage.

Although the implementation of AMLS bears many similarities with the implementation of a block Cholesky factorization, it differs from block Cholesky in terms of the data flow pattern. In particular, the implementation of AMLS cannot be organized as a strictly left-looking or rightlooking procedure. Nonetherless, the major operations in our implementation of AMLS can be conveniently described in terms of the traversal of a separator tree derived from the initial algebraic partitioning of $(K, M)$.

We developed a technique for reducing memory usage in the AMLS calculation. In this technique, only the off-diagonal blocks of $L$ associated with the separator nodes are stored in memory. All other off-diagonal blocks are recomputed whenever they are needed. We demonstrated that such a semi-implicit representation of $L$ can result in up to $50 \%$ savings in memory usage while incuring less than $15 \%$ increase in runtime.

We evaluated our implementation of AMLS on two different applications outside the area of structure analysis. We observed that AMLS is much faster than SIL when a large number of eigenvalues with a few digits of accuracy are of interest. We also observed that both the runtime and memory requirements of AMLS vary with respect to the number of partitioning levels $\left(n_{\text {levels }}\right)$. In particular, the first phase of the AMLS computation, which involves interleaving the congruence transformation of $(K, M)$ with the sub-structure calculation and the projection $(K, M)$ into the subspace assembled from the sub-structure eigenvectors, becomes less time consuming as $n_{\text {levels }}$ increases. However, increasing $n_{\text {levels }}$ tends to increase the dimension of the projection subspace, hence leads to a more costly second phase calculation. More research is needed to identify an optimal choice of $n_{\text {levels }}$. 
A sub-structure mode selection scheme has a large effect on both the accuracy and cost of AMLS computation. We illustrated that the accuracy of the Ritz values may not improve if one simply increases $n_{\text {modes }}$ on either a sub-structure or a separator. However, such an increase in $n_{\text {modes }}$ can lead to a significant increase in AMLS runtime. We asserted that it is more important to select the "important" modes that are associated with the large entries in the eigenvectors $(y)$ of a canonical eigenvalue problem. Although we did not give a recipe for choosing these modes in this paper, our analysis indicates how we might be able to identify these modes by estimating the entries of $y$. We will address the mode selection issue in a separate paper.

\section{References}

[1] K. Bekas and Y. Saad. Computation of smallest eigenvalues using spectral Schur complements. SIAM J. Scientific Computing, 27(2):458-481, 2005.

[2] J. K. Bennighof. Adaptive multi-level substructuring method for acoustic radiation and scattering from complex structures. In A. J. Kalinowski, editor, Computational methods for Fluid/Structure Interaction, volume 178, pages 25-38, AMSE, New York, November, 1993.

[3] J. K. Bennighof and R. B. Lehoucq. An automated multileve substructuring method for eigenspace computation in linear elastodynamics. SIAM Journal on Scientific Computing, $25: 2084-2106,2004$.

[4] R. R. Craig and M. C. C. Bampton. Coupling of substructures for dynamic analysis. AIAA Journal, 6:1313-1319, 1968.

[5] James W. Demmel, Stanley C. Eisenstat, John R. Gilbert, Xiaoye S. Li, and Joseph W. H. Liu. A supernodal approach to sparse partial pivoting. SIAM Journal on Matrix Analysis and Applications, 20(3):720-755, 1999.

[6] K. Elssel and H. Voss. An a priori bound for automated multi-level substructuring. Technical Report 81, Arbeitsbereich Mathematik, TU Hamburg-Harburg, Germany, 2004.

[7] A. George. Nested dissection of a regular finite element mesh. SIAM J. Num. Anal., 10:345$363,1973$.

[8] W. C. Hurty. Vibrations of structure systems by component-mode synthesis. Journal of the Engineering Mechanics Dvision, ASCE, 86:51-69, 1960.

[9] M. F. Kaplan. Implementation of Automated Multilevel Substructuring for Frequency Response Analysis of Structures. PhD thesis, University of Texas at Austin, Austin, TX, December 2001.

[10] G. Karypis and V. Kumar. METIS - A Software Package for Partitioning Unstructured Graphs, Partitioning Meshes, and Computing Fill-Reducing Orderings of Sparse Matrices - Version 4.0. University of Minnesota, 1998.

[11] K. Ko, N. Folwell, L. Ge, A. Guetz, V. Ivanov, L. Lee, Z. Li, I. Malik, W. Mi, C. Ng, and M. Wolf. Electromagnetic systems simulation - from simulation to fabrication. SciDAC report, Stanford Linear Accelerator Center, Menlo Park, CA, 2003. 
[12] A. Kropp and D. Heiserer. Efficient broadband vibro-accoutic analysis of passenger car bodies using an FE-based component mode synthesis approach. J. Computational Acoustics, 11(2):139-157, 2003.

[13] R. B. Lehoucq, D. C. Sorensen, and C. Yang. ARPACK Users' Guide: Solution of Large-Scale Eigenvalue Problems with Implicitly Restarted Arnoldi Methods. SIAM, Philadelphia, 1998.

[14] Esmond G. Ng and Barry W. Peyton. Block sparse Cholesky algorithms on advanced uniprocessor computers. SIAM J. Sci. Comput., 14(5):1034-1056, September 1993.

[15] B. N. Parlett. The Symmetric Eigenvalue Problem. Prentice-Hall, 1980.

[16] C. Yang, W. Gao, Z. Bai, X. Li, L. Lee, P. Husbands, and E. Ng. An algebraic sub-structuring method for large-scale eigenvalue calculation. SIAM J. on Scientific Computing, 27(3):873-892, 2005 .

[17] C. Yang, B. W. Peyton, D. W. Noid, B. G. Sumpter, and R. E. Tuzun. Large-scale normal coordinate analysis for molecular structures. SIAM Journal on Scientific Computing, 23(2):563-582, 2001. 